\title{
Smoking and female sex as key risk factors associated with severe arthralgia in acute and chronic phases of Chikungunya virus infection
}

\author{
IVAN DELGADO-ENCISO ${ }^{1,2}$, BRENDA PAZ-MICHEL $^{3}$, VALERY MELNIKOV ${ }^{1}$, JOSE GUZMAN-ESQUIVEL ${ }^{4}$, \\ FRANCISCO ESPINOZA-GOMEZ ${ }^{1}$, ALEJANDRO D. SORIANO-HERNANDEZ ${ }^{1,2}$, IRAM P. RODRIGUEZ-SANCHEZ ${ }^{5}$, \\ MARGARITA L. MARTINEZ-FIERRO ${ }^{6}{ }$, GABRIEL CEJA-ESPIRITU ${ }^{1,4}$, BERTHA A. OLMEDO-BUENROSTRO ${ }^{1}$, \\ HECTOR R. GALVAN-SALAZAR ${ }^{2,6}$, OSIRIS G. DELGADO-ENCISO ${ }^{7}$, JOSUEL DELGADO-ENCISO ${ }^{7}$, \\ URIEL A. LOPEZ-LEMUS ${ }^{8}$ and DANIEL A. MONTES-GALINDO ${ }^{2}$
}

\author{
${ }^{1}$ School of Medicine, University of Colima, Colima 28030; ${ }^{2}$ Cancerology State Institute, Colima State Health Services, \\ Colima 28085; ${ }^{3}$ Esteripharma México, México City 03100; ${ }^{4}$ Research Unit, IMSS Hospital General de Zona No. 1, \\ Villa de Álvarez, Colima 28983; ${ }^{5}$ Department of Genetics, School of Medicine, Nuevo Leon Autonomous University, \\ Monterrey, Nuevo Leon 64460; ${ }^{6}$ Molecular Medicine Laboratory, Academic Unit of Human Medicine and Health Sciences, \\ Autonomous University of Zacatecas, Zacatecas $98160 ;{ }^{7}$ Ethics Foundation, Study and Cancer Research \\ of The State Institute of Cancerology of Colima; ${ }^{8}$ Department of Health Sciences, \\ Biodefense and Global Infectious Diseases Center, Colima 28000, Mexico
}

Received July 7, 2017; Accepted October 27, 2017

DOI: $10.3892 / \mathrm{etm} .2017 .5668$

\begin{abstract}
Arthralgia is a potentially incapacitating condition and a persistent symptom in chronic or acute episodes of Chikungunya fever caused by infection with the Chikungunya virus (CHIKV). To the best of our knowledge, there are no reports on risk factors associated with the intensity of arthralgias in typical acute episodes of the disease. Although a number of studies have reported on risk factors associated with the development of the chronic stage of the disease, smoking habits have not been analyzed. Smoking is an interesting factor to consider since it is the main environmental risk factor for the development of rheumatoid arthritis (RA), a similar disease to CHIKV in many aspects. In the present study, 140 patients infected with CHIKV were assessed for risk factors associated with severe arthralgia intensity in the acute phase (pain of $9 / 10$ on the visual analog scale of 0-10) and moderate to severe intensity (according to the Routine Assessment of Patient Index Data 3) 3.5 months after infection in patients that experienced the chronic phase of the disease. Women and smokers were 2- to 3-times more likely to experience severe pain in the acute and chronic stages. Likewise, the presence of severe arthralgia during the acute disease phase resulted
\end{abstract}

Correspondence to: Dr Daniel A. Montes-Galindo, Cancerology State Institute, Colima State Health Services, 401 Liceo de Varones Avenue, Colima 28085, Mexico

E-mail: daniel_montes@ucol.mx

Key words: Chikungunya virus, rheumatoid arthritis, risk factor, interleukin-6, arthralgia in a 4-fold increased risk for entering the chronic phase. Smoking was a more important risk factor in males compared with females. Smoking resulted in a 20-fold increased risk for severe arthralgia during the acute phase in men, as well as a 10-fold increased risk for developing chronic disease with moderate-to-severe pain 3.5 months after the acute stage. The presence of rash, headache, muscular weakness or conjunctivitis in the acute phase, the presence of diabetes and age $>40$ years were considered significant risk factors due to their influence on illness progression. In conclusion, smoking and female sex were the main risk factors associated with development of severe joint pain in the acute and chronic phases of Chikungunya fever. These risk factors are similar to those associated with the development and severity of RA, possibly because the two diseases share pathophysiological mechanisms, including elevated interleukin- 6 levels.

\section{Introduction}

Chikungunya fever is a disease caused by the Chikungunya virus (CHIKV), an RNA virus from the genus Alfavirus (1). CHIKV is transmitted to humans through the bite of infected Aedes aegypti and Aedes albopictus mosquitoes (2). These species are prevalent in tropical and subtropical regions, with the potential to affect over one billion people (3). CHIKV infection can present at acute, sub-acute or chronic phase. The acute phase is characterized by a complete disappearance of all symptoms by day 10 from symptom onset, whereas the chronic phase is characterized by symptom persistence of $>3$ months (4). Symptoms start abruptly in the acute stage of the disease, with high fever $(85-100 \%)$ and arthralgia (90-98\%) that is often disabling (5). Other symptoms include 
headache, muscular weakness, arthritis and dermatological signs (5), as well as digestive disorders, including nausea and vomiting $(1-3,5)$. In the chronic stage, joint inflammation and arthralgia persists. A previous study on 39 patients with chronic CHIKV infection documented a prevalence of chronic inflammatory polyarthralgia in up to $89 \%$ of cases (6). In a study conducted in western Mexico, the researchers reported persistent arthralgia in $62.5 \%$ of the patients, 3 months after infection. Symptoms prevailed in $42 \%$ of these patients at 6 months after infection, whereas they were attenuated in other patients with the passage of time (7).

Studies searching for an association or correlation between molecular, clinical or sociodemographic characteristics and disease severity of CHIKV disease are limited. To the best of our knowledge, only one study has been conducted looking for risk factors associated with illness severity in atypical cases of acute CHIKV disease in adults. The authors identified that hypertension and underlying respiratory or cardiac conditions were independent risk factors for disease severity (8). On the other hand, several studies have identified risk factors associated with the chronic phase of the disease. A recent systematic review analyzed 37 studies and concluded that female sex, older age and high levels of interleukin-6 (IL-6), as well as the severity of the acute phase and certain co-morbidities, were associated with persistent arthralgia (9).

Potential risk factors, including smoking habits, have not been considered in association with CHIKV infection. The study of smoking habits in patients with this disease is of interest, since smoking is one of the most important risk factors known and studied for the development of rheumatoid arthritis (RA). Chronic Chikungunya arthritis symptoms closely mimic RA symptoms and even share certain molecular biomarkers (10), including high levels of IL-6, (11,12), so it can be assumed that the two pathologies may also share key risk factors. The identification of possible similarities between CHIKV arthritis and RA would provide a basis for identifying the pathophysiological and therapeutic molecular mechanisms of the two diseases. The present study searched for risk factors associated with the development of very severe arthralgia in acute and chronic phases of CHIKV infection, with an emphasis on the analysis of smoking habits.

\section{Materials and methods}

Ethical approval. This project was approved by the Research Ethics Committee of the Cancerology State Institute, Colima State Health Services (Colima, Mexico) and all participants signed statements of informed consent. Participation in the study was voluntary.

Patient data. The present study included patients with acute CHIKV fever that were admitted to any of the four hospitals of the Health Department of the State of Colima (western region of the Mexican Pacific coast), from July to October 2015. In the acute phase of the disease, patients were evaluated by hospital staff physicians, who filled out standardized clinical records for an epidemiologic study of vector-borne diseases. Personal data, including telephone numbers, and the absence or presence of clinical manifestations, including fever, nausea, vomiting, itching, conjunctivitis, myalgia and arthralgia, were recorded. Intensity of joint pain was recorded according to the visual analog scale (VAS), from 'no pain' (score of 0) to 'worst imaginable plain' (score of 10) (13). VAS was selected because it is currently the validated scale that best evaluates pain in diseases that manifest arthralgia, including RA (13). Patient's blood samples were sent to the Public Health Laboratory of the Health Department of the State of Colima (Colima, Mexico) for serum analysis and CHIKV diagnostic by using the Trioplex Real-time RT-PCR Assay (Biosystems ${ }^{\mathrm{TM}}$ TaqMan $^{\mathrm{TM}}$ Arbovirus Triplex Assay; Thermo Fisher Scientific, Inc., Waltham, MA, USA), according to manufacturer's instructions for detection and differentiation of RNA from dengue, chikungunya or zika (14).

The study sample included 140 patients $\geq 18$ years old (average age, $39.9 \pm 14.5 ; 71.4 \%$ women) who were positive for CHIKV through RT-qPCR evaluation and provided clinical and personal data during their acute phase, as described above. The patients were also required to be available for a telephone interview 3.5 months after acute infection (chronic phase evaluation). Patients with a self-reported history of systemic rheumatologic disease (RA, multiple sclerosis or systemic lupus erythematosus) were excluded from the analysis. Evaluation of the chronic phase was performed according to the Routine Assessment of Patient Index Data 3 (RAPID3), which is a pooled index of the three patient-reported RA Core Data Set measures of the American College of Rheumatology: Physical function, pain and patient estimate of global status (15). Each of the three individual measures was scored from 0 to 10 , for a total of 30 points. Disease severity scores were classified as follows: RAPID3 score, $>12=$ high; $>6$ and $\leq 12=$ moderate; $>3$ and $\leq 6=$ low; $\leq 3=$ remission. Previous findings have correlated RAPID3 scores with the Disease Activity Score 28 and the Clinical Disease Activity Index (15). Additionally, patients were required to provide their most intense arthralgia value (VAS 0-10) within the past $24 \mathrm{~h}$, by telephone, which was set as the arthralgia score for the chronic stage of disease.

Pain evaluation. Pain stratification is a complex topic. Previous studies have attempted to identify pain intensity cutoff points using diverse measuring scales (16-18). However, it has been determined that cutoff points vary, depending on pain type, chronicity, diagnosis, age, co-morbid conditions, cultures and the interference of pain with various activities (including enjoyment of life, daily activity, walking, mood, sleep and work) (19). Thus, VAS scores for pain for classifying patients with severe or very severe pain have varied in different studies (19). In the present study, patients were divided according to their pain in the acute phase, using 9 as the cutoff point (50th percentile of the VAS score). Patients with low arthralgia scores ( $\leq 8$ points) during the acute phase formed the reference group, while patients with higher scores ( 9 and 10) were considered the study group. The score of 9-10 has been used in previous studies to classify patients with very severe pain or the most severe pain imaginable $(20,21)$. In the chronic phase of the disease, the patients with RAPID3 scores $\leq 6$ formed the reference group, whereas patients with scores $>6$ (moderate/high disease activity) were considered the study group.

Smoking habits. Smoking habits were also evaluated using a definition similar to that established by the CDC National 
Health Interview Survey (22). The 100-cigarette threshold has been extensively used to pragmatically identify lifetime smokers (current or former smokers) and differentiate them from experimental or new-onset smokers (23). This classification was also used to evaluate the effect of smoking on the severity of a systemic viral infection causing renal damage (24). In the present study, current smokers were defined as patients that had smoked $\geq 100$ cigarettes in their lifetime and that currently smoked daily or occasionally. Ex-smokers were defined as patients that had smoked $\geq 100$ cigarettes in their lifetime, but that had not smoked daily or occasionally for $\geq 1$ year. Never-smokers were defined as patients that had smoked $<100$ cigarettes in their lifetime. According to the questionnaire, the patients were divided into 2 groups to evaluate the effect of current smoking on the severity of acute and chronic phases of CHIKV infection: Current smokers and non-smokers. The group of non-smokers included the ex-smokers and never-smokers. No patients changed their smoking habits during the study.

Statistical analysis. Data are presented as the mean \pm standard deviation. For the inferential statistics, normal data distribution was first determined using the Kolmogorov-Smirnov test and the equality of variances was confirmed using Levene's test. Student's t-test was used to compare the mean values of the normally distributed measurement variables and the homogeneity of variances. Data with non-normal distribution or non-homogeneous variances were evaluated using the Mann-Whitney U test, and groups were compared using the $\chi^{2}$ test. The association between symptoms in the acute phase of the disease (including headache, rash and muscular weakness), or factors such as sex or smoking habits, and intense arthralgia in the acute (VAS 9/10) or chronic (RAPID3 score, $>6$ ) phase, were estimated by odds ratios (OR) and 95\% confidence intervals (CI), using the Crosstabs procedure. The risk factors of sex, smoking and age $>40$ years were adjusted through a Cochran-Mantel-Haenszel analysis. For the statistical analysis of disease severity levels obtained from the RAPID3 index, the level of severity was transformed into a numerical value (ordinal scale), where remission $=0$, mild disease $=1$, moderate disease $=2$ and severe disease $=3$. $\mathrm{P}<0.05$ was considered to indicate a statistically significant difference. All statistical analyses were performed with SPSS version 20 software (IBM Corp., Armonk, NY, USA).

\section{Results}

Patient characteristics during acute phase. In total, 53\% of patients presented with intense arthralgia during the acute phase of the disease, classified as those with 9-10 points on the VAS. The mean VAS score in the intense arthralgia group was $9.7 \pm 0.46$, compared with a mean score of $7.0 \pm 1.4$ points in the control group. As shown in Table I, females, smokers and patients who were $\geq 40$ years old exhibited an increased risk of experiencing high-intensity arthralgia during the acute phase of the disease. This was observed to be a significant increase for female sex (adjusted $\mathrm{P}=0.03$ ) and smoking (adjusted $\mathrm{P}=0.02)$ However, the increased risk for patients who were $\geq 40$ years old was not observed to be statistically significant when adjusted for sex and smoking habits.
Patient characteristics during chronic phase. A notable finding was that 3.5 months after CHIKV infection, $68 \%$ of the patients had entered either a moderate or severe chronic stage of the disease, according to the RAPID3 multidimensional health assessment questionnaire. For patients with chronic disease, the mean VAS score for arthralgia was 5.7 \pm 2.8 , compared with $0.9 \pm 1.4$ for the control group (patients with RAPID3 values $\leq 6)(\mathrm{P}<0.001)$. A total of $78 \%$ of patients with moderate/severe chronic disease and $24 \%$ of patients with mild chronic disease, or in remission, used non-steroidal anti-inflammatory drugs (NSAIDs) for pain relief. Paracetamol was the most widely prescribed pain relief medication among all participants (70\%). Anti-rheumatic drugs were not registered for any of the patients of the present study.

Risk factors for entering chronic phase. As indicated in Table II, female sex, smoking and the presence of severe arthralgia in the acute phase of the Chikungunya fever were risk factors associated with developing a severe chronic stage. Statistical adjustment of the data (for sex and smoking) confirmed these risk factors (female, adjusted $\mathrm{P}=0.005$; smoking, adjusted $\mathrm{P}=0.03$; severe arthralgia in acute phase, $\mathrm{P}=0.002$ ). The adjusted analysis also demonstrated that the presence of exanthema during the acute phase of disease increased the risk of entering chronic stage (adjusted $\mathrm{P}=0.02$ ), whereas conjunctivitis reduced the risk of entering chronic stage (adjusted $\mathrm{P}=0.04$ ).

Risk factors for severe pain. Table III indicates the mean values for arthralgia intensities (VAS scores) in patients during the acute and chronic stages of Chikungunya fever, as well as the RAPID3 level of chronic disease severity, according to the presence or absence of certain clinical characteristics in the acute phase of the disease. It was evident that patients that smoked, as well as patients of the female sex, experienced significantly higher pain intensity during the acute phase of disease $(\mathrm{P}=0.003)$. Likewise, patients with severe arthralgia in the acute phase experienced significantly higher arthralgia 3.5 months after infection $(\mathrm{P}=0.001)$, as well as more severe chronic disease $(\mathrm{P}<0.001)$. Patients $\geq 40$ years of age did not exhibit a significant increase in pain during the acute phase of the disease or in severity of chronic disease, but did exhibit a significant increase in pain during the chronic stage $(\mathrm{P}=0.004)$. Patients with exanthema in the acute phase did not experience a significant increase in pain intensity during the chronic stage, despite what was observed in Table II. The presence of conjunctivitis was associated with a significant decrease in the severity of chronic disease $(\mathrm{P}=0.04)$. However, patients with conjunctivitis did not exhibit a significant reduction in pain intensity $(\mathrm{P}=0.58)$.

Patients that experienced headache and muscular weakness during the acute phase also presented, with significantly more intense, arthralgia in the acute and chronic stages. Furthermore, the severity of chronic disease was significantly higher in patients that experienced headache $(\mathrm{P}=0.02$; Table III). In patients with diabetes, arthralgia was significantly more intense during the chronic stage.

Hormonal status of female patients did not significantly modify the risk of experiencing intense pain during the acute phase $(\mathrm{OR}, 1.5 ; 95 \% \mathrm{CI}, 0.6-3.7 ; \mathrm{P}=0.22)$ or severe chronic 
Table I.Association between clinical characteristics of patients and high-intensity arthralgia during the acute phase of Chikungunya infection.

\begin{tabular}{|c|c|c|c|c|c|c|}
\hline Characteristic & $\begin{array}{l}\text { Controls, } \\
\%(n=66)\end{array}$ & $\begin{array}{c}\text { Cases, } \\
\%(n=74)\end{array}$ & $\begin{array}{c}\text { Crude OR } \\
(95 \% \mathrm{CI})\end{array}$ & P-value & $\begin{array}{c}\text { Adjusted } \\
\mathrm{OR}^{\mathrm{a}}(95 \% \mathrm{CI})\end{array}$ & $\begin{array}{c}\text { Adjusted } \\
\text { P-value }\end{array}$ \\
\hline Smoking & 13.6 & 35.1 & $3.43(1.46-8.02)$ & $0.004^{\mathrm{b}}$ & 3.03 (1.16-7.86) & $0.020^{\mathrm{b}}$ \\
\hline Female sex & 62.1 & 79.7 & $2.30(1.12-5.09)$ & $0.020^{\mathrm{b}}$ & $2.42(1.04-5.58)$ & $0.030^{\mathrm{b}}$ \\
\hline Age $\geq 40$ years & 42.4 & 59.4 & $1.99(1.01-3.90)$ & $0.040^{\mathrm{b}}$ & $1.44(0.67-3.10)$ & 0.340 \\
\hline Diabetes & 10.6 & 12.1 & $1.16(0.40-3.33)$ & 0.770 & $0.52(0.11-2.34)$ & 0.390 \\
\hline Obesity & 30.3 & 39.1 & $1.48(0.73-2.99)$ & 0.270 & $1.32(0.58-3.00)$ & 0.580 \\
\hline Hypertension & 19.6 & 12.1 & $0.56(0.22-1.42)$ & 0.220 & $0.36(0.12-1.05)$ & 0.060 \\
\hline Exanthema & 45.4 & 45.9 & $1.00(0.52-1.98)$ & 0.950 & $1.55(0.68-3.52)$ & 0.290 \\
\hline Conjunctivitis & 9.0 & 12.1 & $1.38(0.46-4.12)$ & 0.580 & $1.55(0.41-5.75)$ & 0.510 \\
\hline Pruritus & 24.2 & 25.6 & $1.07(0.50-2.32)$ & 0.840 & $1.35(0.52-3.49)$ & 0.530 \\
\hline Muscular weakness & 1.5 & 9.4 & $6.79(0.81-56.74)$ & 0.070 & $3.70(0.39-35.17)$ & 0.250 \\
\hline Headache & 93.3 & 98.6 & $4.70(0.51-43.24)$ & 0.170 & $2.50(0.26-25.34)$ & 0.410 \\
\hline Retroocular pain & 63.6 & 60.8 & $0.88(0.44-1.75)$ & 0.730 & $0.74(0.32-1.66)$ & 0.460 \\
\hline Photophobia & 19.6 & 17.5 & $0.86(0.37-2.03)$ & 0.740 & $1.42(0.49-4.06)$ & 0.510 \\
\hline Nasal congestion & 1.5 & 6.7 & $4.70(0.53-41.40)$ & 0.160 & ND & \\
\hline Arthritis & 48.4 & 40.5 & $0.72(0.37-1.41)$ & 0.340 & $0.67(0.30-1.50)$ & 0.330 \\
\hline Vomiting & 13.6 & 9.4 & $0.66(0.23-1.88)$ & 0.440 & $0.60(0.19-1.93)$ & 0.400 \\
\hline Nausea & 28.7 & 37.8 & $1.50(0.74-3.06)$ & 0.250 & $1.71(0.73-3.9)$ & 0.210 \\
\hline Diarrhea & 10.6 & 4.0 & $0.35(0.08-1.43)$ & 0.140 & $0.57(0.11-2.99)$ & 0.510 \\
\hline Abdominal pain & 16.6 & 10.8 & $0.60(0.22-1.61)$ & 0.310 & $0.62(0.18-2.16)$ & 0.450 \\
\hline Dysgeusia & 10.6 & 13.5 & $1.31(0.47-3.68)$ & 0.320 & $2.23(0.60-8.25)$ & 0.220 \\
\hline
\end{tabular}

Symptoms are those suffered by patients in the acute phase. P-values were calculated using the $\chi^{2}$ test. ${ }^{a}$ Adjusted for the variables of smoking (yes/no), sex (male/female) and age $\geq 40$ years (yes/no) through the Cochran-Mantel-Haenszel method. In a case, data were considered ND due to very small or lack of data for different strata of adjusted analysis. ${ }^{b} \mathrm{P}<0.05$. OR, odds ratio; CI, confidence intervals; ND, not determined.

disease (OR, 1.6; 95\% CI, 0.5-4.7; P=0.25), as observed from patients with menopause (data not shown).

Effects of smoking habits. Smoking habits modified the course of the disease differently in men and women. As indicated in Table IV, smoking increased pain intensity in the acute and chronic stages of the disease in males and females. However, this increase was only observed to be significant in males $(\mathrm{P}<0.001$ at acute stage; $\mathrm{P}=0.003$ at chronic stage). Arthralgia scores during the acute or chronic stage of the infection were similar among male and female smokers. However, in male and female non-smokers, males exhibited a significantly lower risk of experiencing arthralgia at the acute and chronic stages (both $\mathrm{P}<0.001)$. Mean arthralgia values in the chronic phase for male non-smokers were $62 \%$ less intense compared with male smokers. On the other hand, pain intensity was reduced by only $15 \%$ in female non-smokers compared with female smokers. These data indicate that smoking, in relation to arthralgia intensity in CHIKV infection, affected males to a greater extent than females, and that arthralgia pain intensity was generally stronger in male smokers. Male smokers exhibited a 20 -fold increased risk for experiencing very intense pain during the acute phase (OR, 20.1 95\% CI, 2.1-189.8; $\mathrm{P}=0.003)$, and a 10 -fold increased risk for entering a moderate/severe chronic phase after 3.5 months, compared with non-smokers (OR, 10.5; 95\% CI, 1.1-96.5; P=0.02).
In agreement with these results, severity of arthritis due to CHIKV infection was significantly increased by smoking habits, according to RAPID 3 scores, which was only indicated in male smokers $(\mathrm{P}=0.04$, Table IV).

\section{Discussion}

Smoking and female sex are the key risk factors for experiencing severe arthralgia during the acute and chronic phases of CHIKV infection. There are no previous reports associating smoking habits with the severity of symptoms during CHIKV infection, but it is logical to assume that smoking can influence the severity of a viral infection that affects the lungs, since they could already be compromised by the chemical components of cigarette smoke. This premise has previously been demonstrated in association with influenza infection (25). Smoking has also been demonstrated to influence the severity of viral infections in other organs. For example, smoking has recently been reported to be associated with aggravated kidney damage in Puumala hantavirus-induced hemorrhagic fever with renal syndrome (24).

Smoking can also influence viral pathogenesis in numerous ways. For instance, viral replication of the respiratory syncytial virus has been reported to increase in cell cultures previously exposed to cigarette smoke extract (26). Additionally, cigarette smoke reduces the expression of interferon-inducible genes, 
Table II. Association between clinical characteristics of patients and moderate/severe disease in the chronic phase of Chikungunya infection.

\begin{tabular}{lcccccc}
\hline Characteristic & $\begin{array}{c}\text { Controls, } \\
\%(\mathrm{n}=45)\end{array}$ & $\begin{array}{c}\text { Cases, } \\
\text { (n=95) }\end{array}$ & $\begin{array}{c}\text { Crude OR } \\
(95 \% \mathrm{CI})\end{array}$ & $\begin{array}{c}\text { P-value } \\
\text { Adjusted OR }{ }^{\mathrm{a}} \\
(95 \% \mathrm{CI})\end{array}$ & $\begin{array}{c}\text { Adjusted } \\
\text { P-value }\end{array}$ \\
\hline Smoking & 13.3 & 30.5 & $2.85(1.08-7.48)$ & $0.030^{\mathrm{c}}$ & $2.93(1.05-8.14)$ & $0.030^{\mathrm{c}}$ \\
Female sex & 55.5 & 78.9 & $3.0(1.39-6.46)$ & $0.005^{\mathrm{c}}$ & $3.13(1.40-6.98)$ & $0.005^{\mathrm{c}}$ \\
$\geq 40$ years & 44.4 & 54.7 & $1.51(0.74-3.08)$ & 0.250 & $1.51(0.70-3.23)$ & 0.280 \\
Severe arthralgia $^{\mathrm{b}}$ & 28.8 & 64.2 & $4.41(2.04-9.52)$ & $<0.001^{\mathrm{c}}$ & $3.65(1.57-8.44)$ & $0.002^{\mathrm{c}}$ \\
Diabetes & 8.8 & 12.6 & $1.48(0.45-4.88)$ & 0.510 & $1.43(0.36-5.63)$ & 0.600 \\
Obesity & 26.6 & 38.9 & $1.75(0.80-3.82)$ & 0.150 & $1.57(0.0 .67-3.67)$ & 0.290 \\
Hypertension & 11.1 & 17.8 & $1.74(0.59-5.07)$ & 0.300 & $1.36(0.46-4.02)$ & 0.570 \\
Exanthema & 33.3 & 51.5 & $2.01(0.96-4.22)$ & 0.060 & $3.07(1.17-8.03)$ & $0.020^{\mathrm{c}}$ \\
Conjunctivitis & 17.7 & 7.3 & $0.36(0.12-1.08)$ & 0.070 & $0.26(0.07-0.94)$ & $0.040^{\mathrm{c}}$ \\
Pruritus & 17.7 & 28.4 & $1.83(0.75-4.44)$ & 0.170 & $2.88(0.89-9.29)$ & 0.070 \\
Muscular weakness & 2.2 & 7.3 & $3.50(0.41-29.34)$ & 0.240 & $\mathrm{ND}$ & \\
Headache & 93.3 & 97.8 & $3.32(0.53-20.62$ & 0.190 & $7.32(0.54-98.11)$ & 0.130 \\
Retroocular pain & 66.6 & 60.0 & $0.75(0.35-1.57)$ & 0.440 & $0.66(0.27-1.59)$ & 0.350 \\
Photophobia & 24.4 & 15.7 & $0.57(0.24-1.39)$ & 0.220 & $0.91(0.32-2.56)$ & 0.860 \\
Nasal congestion & 2.2 & 5.2 & $2.44(0.27-21.56)$ & 0.420 & ND & \\
Arthritis & 44.4 & 44.2 & $0.99(0.48-2.02)$ & 0.970 & $0.78(0.33-1.86)$ & 0.580 \\
Vomiting & 13.3 & 10.5 & $0.76(0.25-2.25)$ & 0.620 & $0.41(0.11-1.48)$ & 0.170 \\
Nausea & 28.8 & 36.8 & $1.43(0.66-3.09)$ & 0.350 & $1.76(0.69-4.49)$ & 0.230 \\
Diahrrea & 4.4 & 7.3 & $1.71(0.34-8.58)$ & 0.510 & $2.30(0.43-12.14)$ & 0.320 \\
Abdominal pain & 8.8 & 15.7 & $1.92(0.59-6.16)$ & 0.270 & $1.72(0.42-6.98)$ & 0.440 \\
Dysgeusia & 15.5 & 10.52 & $0.63(0.22-1.80)$ & 0.390 & $0.78(0.22-2.72)$ & 0.700 \\
\hline
\end{tabular}

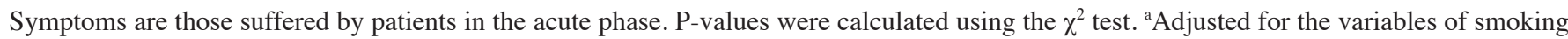
(yes/no) and sex (male/female) through the Cochran-Mantel-Haenszel method. 'Severe arthralgia (visual analog scale 9-10) during acute phase. ${ }^{\mathrm{c}} \mathrm{P}<0.05$. OR, odds ratio; $\mathrm{CI}$, confidence intervals. In some cases, data were considered ND due to very small or lack of data for different strata of adjusted analysis. ND, not determined.

in turn, limiting the antiviral responses, in a neonatal mouse model (27). This evidence is consistent with the increased severity of CHIKV infection observed in patients that smoked in the present study, particularly regarding arthralgia intensity in the acute phase of illness.

IL- 6 has been demonstrated to be involved in the pathophysiology of CHIKV infection. Increased IL- 6 levels are associated with increased severity and persistence of joint pain (4). Notably, cigarette smoke extract in non-cytotoxic doses is capable of increasing the levels of IL-6 in cell cultures (28) and in animal models (29). Additionally, smoking correlates with higher serum levels of IL-6 in apparently healthy individuals, the elderly and HIV-infected patients (30-32). An increase in pre-existing IL-6 levels in smokers could be an important cause of symptom severity in smokers that acquire CHIKV infection. Smoking has been observed to affect the immune response at the cellular and humoral level, hence it could induce proinflammatory and immunosuppressive effects (33). Thus, additional immunological alterations may be producing general and severe symptoms in patients that are smokers.

It is widely established that chronic sequelae of CHIKV infection mimic RA (34). For example, high levels of IL-6 are associated with inflammation and joint damage in RA patients (35). Thus, the two pathologies share a clinical profile and high levels of IL-6. In addition, smoking is the main environmental risk factor for developing RA and is also associated with promoting early stages and higher severity of the disease. For these reasons, in the present study, it was investigated whether smoking is a shared risk factor with Chikungunya infection arthralgia. Notably, serum levels of ILs (including IL-6) in ex-smokers have been reported to not be significantly reduced, even 4 months after quitting smoking (36). Therefore, the severity of arthralgia caused by CHIKV infection in smokers may not disappear until many months after quitting. This requires further exploration in future investigations.

To the best of our knowledge, no previous studies have established an association between arthralgia intensity during the acute phase of typical CHIKV infection and the subsequent symptomatology in patients that progress into chronic stage. Nevertheless, previous reports associate many risk factors with arthralgia persistence. A recent systematic review analyzed 37 studies and concluded that female sex, older age and high levels of IL-6, as well as the severity of the acute phase and some co-morbidities, were associated with persistent arthralgia (37). These clinical results are consistent with those reported in the present study: Female sex as a risk factor for experiencing high-intensity arthralgia during the acute and chronic stages of the disease; a strong tendency for both 
Table III. Arthralgia intensity in the acute and chronic phases of Chikungunya infection, according to the absence or presence of clinical characteristics.

\begin{tabular}{|c|c|c|c|c|c|c|c|c|c|}
\hline \multirow{2}{*}{$\begin{array}{l}\text { Clinical } \\
\text { characteristic }\end{array}$} & \multicolumn{2}{|c|}{$\begin{array}{l}\text { VAS score of arthralgia } \\
\text { during acute phase }\end{array}$} & \multirow[b]{2}{*}{ P-value } & \multicolumn{2}{|c|}{$\begin{array}{l}\text { VAS score of arthralgia } \\
\text { during chronic phase }\end{array}$} & \multirow[b]{2}{*}{ P-value } & \multicolumn{2}{|c|}{$\begin{array}{l}\text { RAPID3 score of } \\
\text { chronic disease severity }\end{array}$} & \multirow[b]{2}{*}{ P-value } \\
\hline & Presence & Absence & & Presence & Absence & & Presence & Absence & \\
\hline Smoking & $9.1 \pm 1.3$ & $8.2 \pm 1.7$ & $0.003^{\mathrm{b}}$ & $5.4 \pm 3.0$ & $3.9 \pm 3.3$ & $0.020^{\mathrm{b}}$ & $2.4 \pm 0.9$ & $1.8 \pm 1.3$ & $0.040^{\mathrm{b}}$ \\
\hline Female & $8.7 \pm 1.4$ & $7.6 \pm 2.0$ & $0.003^{\mathrm{b}}$ & $4.8 \pm 3.1$ & $2.9 \pm 3.3$ & $0.003^{\mathrm{b}}$ & $2.2 \pm 1.2$ & $1.4 \pm 1.3$ & $<0.001^{\mathrm{b}}$ \\
\hline Age $\geq 40$ years & $8.5 \pm 1.7$ & $8.1 \pm 1.6$ & 0.070 & $5.2 \pm 3.0$ & $3.5 \pm 3.3$ & $0.004^{b}$ & $2.2 \pm 1.2$ & $1.8 \pm 1.3$ & 0.070 \\
\hline Severe arthralgia ${ }^{a}$ & $9.7 \pm 0.4$ & $7.0 \pm 1.4$ & $<0.001^{\mathrm{b}}$ & $5.1 \pm 3.1$ & $3.2 \pm 3.3$ & $0.001^{\mathrm{b}}$ & $2.4 \pm 1.0$ & $1.5 \pm 1.3$ & $<0.001^{\mathrm{b}}$ \\
\hline Diabetes & $8.4 \pm 2.0$ & $8.4 \pm 1.6$ & 0.560 & $6.2 \pm 3.3$ & $4.0 \pm 3.2$ & $0.020^{\mathrm{b}}$ & $2.5 \pm 0.9$ & $1.9 \pm 1.3$ & 0.170 \\
\hline Obesity & $8.5 \pm 1.6$ & $8.3 \pm 1.7$ & 0.440 & $4.6 \pm 3.0$ & $4.1 \pm 3.4$ & 0.400 & $2.2 \pm 1.2$ & $1.8 \pm 1.3$ & 0.140 \\
\hline Hypertension & $8.3 \pm 1.7$ & $8.4 \pm 1.6$ & 0.770 & $5.2 \pm 3.3$ & $4.1 \pm 3.3$ & 0.150 & $2.3 \pm 1.0$ & $1.9 \pm 1.3$ & 0.170 \\
\hline Exanthema & $8.4 \pm 1.4$ & $8.4 \pm 1.9$ & 0.540 & $4.7 \pm 3.4$ & $4.4 \pm 3.2$ & 0.680 & $2.2 \pm 1.1$ & $2.0 \pm 1.3$ & 0.670 \\
\hline Conjunctivitis & $8.1 \pm 2.5$ & $8.4 \pm 1.6$ & 0.750 & $4.0 \pm 3.4$ & $4.6 \pm 3.2$ & 0.580 & $1.5 \pm 1.3$ & $2.2 \pm 1.2$ & 0.040 \\
\hline Pruritus & $8.4 \pm 1.6$ & $8.4 \pm 1.7$ & 0.910 & $5.3 \pm 3.1$ & $4.3 \pm 3.3$ & 0.150 & $2.4 \pm 1.1$ & $2.0 \pm 1.2$ & 0.140 \\
\hline Muscular weakness & $9.5 \pm 0.7$ & $8.3 \pm 1.7$ & $0.040^{\mathrm{b}}$ & $8.4 \pm 1.5$ & $4.2 \pm 3.2$ & $0.001^{\mathrm{b}}$ & $2.7 \pm 0.6$ & $2.0 \pm 1.2$ & 0.080 \\
\hline Headache & $8.5 \pm 1.5$ & $6.2 \pm 3.0$ & 0.050 & $4.4 \pm 3.2$ & $1.8 \pm 2.4$ & 0.070 & $2.0 \pm 1.2$ & $0.6 \pm 1.3$ & $0.020^{\mathrm{b}}$ \\
\hline Photophobia & $8.2 \pm 2.1$ & $8.4 \pm 1.6$ & 0.970 & $4.1 \pm 3.1$ & $4.6 \pm 3.3$ & 0.530 & $1.9 \pm 0.3$ & $1.2 \pm 1.1$ & 0.770 \\
\hline Retroocular pain & $8.5 \pm 1.5$ & $8.2 \pm 2.0$ & 0.610 & $4.7 \pm 3.2$ & $4.2 \pm 3.3$ & 0.380 & $2.0 \pm 1.2$ & $2.1 \pm 1.3$ & 0.680 \\
\hline Nasal congestion & $9.5 \pm 0.5$ & $8.3 \pm 1.7$ & 0.190 & $7.2 \pm 1.2$ & $4.4 \pm 3.3$ & 0.090 & $2.5 \pm 0.5$ & $2.0 \pm 1.2$ & 0.910 \\
\hline Arthritis & $8.4 \pm 1.7$ & $8.3 \pm 1.6$ & 0.640 & $4.3 \pm 3.2$ & $4.7 \pm 3.3$ & 0.560 & $2.0 \pm 1.2$ & $2.1 \pm 1.2$ & 0.720 \\
\hline Vomiting & $8.2 \pm 1.8$ & $8.4 \pm 1.7$ & 0.840 & $5.0 \pm 3.2$ & $4.4 \pm 3.2$ & 0.520 & $1.9 \pm 1.1$ & $2.1 \pm 1.2$ & 0.400 \\
\hline Nausea & $8.6 \pm 1.6$ & $8.3 \pm 1.7$ & 0.350 & $4.6 \pm 3.2$ & $4.4 \pm 3.3$ & 0.800 & $2.2 \pm 1.1$ & $2.0 \pm 1.2$ & 0.470 \\
\hline Diarrhea & $7.3 \pm 2.5$ & $8.5 \pm 1.6$ & 0.180 & $3.8 \pm 3.7$ & $4.6 \pm 3.2$ & 0.560 & $2.4 \pm 1.1$ & $2.0 \pm 1.2$ & 0.340 \\
\hline Abdominal pain & $8.2 \pm 1.6$ & $8.4 \pm 1.7$ & 0.540 & $5.2 \pm 3.2$ & $4.4 \pm 3.2$ & 0.350 & $2.5 \pm 1.0$ & $2.0 \pm 1.2$ & 0.120 \\
\hline Dysgeusia & $8.4 \pm 2.2$ & $8.4 \pm 1.6$ & 0.520 & $4.3 \pm 3.4$ & $4.5 \pm 3.2$ & 0.710 & $1.8 \pm 1.4$ & $2.1 \pm 1.2$ & 0.580 \\
\hline
\end{tabular}

Data are presented as the mean \pm standard deviation. Arthralgia was quantified using VAS scores, from 'no pain' (score of 0) to 'worst pain imaginable' (score of 10). Severity chronic disease was quantified using the RAPID3 index by transforming the severity level into a

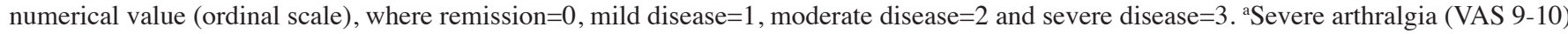
during acute phase. P-values were calculated by performing the Mann-Whitney U test. ${ }^{b} \mathrm{P}<0.05$. VAS, visual analog scale; RAPID3, Routine Assessment of Patient Index Data 3.

Table IV. Arthralgia intensity in the acute and chronic phases of Chikungunya infection, according to the sex and smoking habits of patients.

\begin{tabular}{|c|c|c|c|c|c|c|c|c|c|}
\hline \multirow[b]{2}{*}{ Sex } & \multicolumn{2}{|c|}{$\begin{array}{l}\text { VAS score of arthralgia } \\
\text { during acute phase }\end{array}$} & \multirow[b]{2}{*}{ P-value } & \multicolumn{2}{|c|}{$\begin{array}{l}\text { VAS score of arthralgia } \\
\text { during chronic phase }\end{array}$} & \multirow[b]{2}{*}{ P-value } & \multicolumn{2}{|c|}{$\begin{array}{l}\text { RAPID3 score of } \\
\text { chronic disease severity }\end{array}$} & \multirow[b]{2}{*}{ P-value } \\
\hline & Smoker & Non-smoker & & Smoker & Non-smoker & & Smoker & Non-smoker & \\
\hline Male $n=40$ & $9.2 \pm 0.9$ & $7.2 \pm 2.0$ & $<0.001^{\mathrm{a}}$ & $5.6 \pm 3.4$ & $2.1 \pm 2.8$ & $0.003^{\mathrm{a}}$ & $2.1 \pm 0.9$ & $1.2 \pm 1.3$ & $0.040^{\mathrm{a}}$ \\
\hline Female $n=100$ & $9.0 \pm 1.3$ & $8.6 \pm 1.3$ & 0.080 & $5.4 \pm 2.7$ & $4.6 \pm 3.2$ & 0.140 & $2.4 \pm 0.9$ & $2.1 \pm 1.2$ & 0.110 \\
\hline P-value & 0.350 & $<0.001^{\mathrm{a}}$ & & 0.440 & $<0.001^{\mathrm{a}}$ & & 0.200 & $<0.001^{\mathrm{a}}$ & \\
\hline
\end{tabular}

Data are presented as the mean \pm standard deviation. Arthralgia was quantified using VAS scores, from 'no pain' (score of 0) to 'worst pain imaginable' (score of 10). Severity of chronic disease was quantified using the RAPID3 index by transforming the severity level into a numerical value (ordinal scale), where remission $=0$, mild disease $=1$, moderate disease $=2$ and severe disease $=3$. $P$-values were calculated by performing the Mann-Whitney U test. ${ }^{a} \mathrm{P}<0.05$. VAS, visual analog scale; RAPID3, Routine Assessment of Patient Index Data 3.

sexes to go into a moderate or severe chronic stage if there was intense arthralgia during the acute phase; and an increased risk for high-intensity arthralgia during the chronic stage in patients $\geq 40$ years of age. 
According to results observed in the present study, for Chikungunya fever in general, female sex and smoking were the most important risk factors that induced a disease with severe symptomatology. This trend is also observed in rheumatoid arthritis, with females exhibiting worse progression compared with males (38). The inflammatory response has been demonstrated to differ between the sexes. A clinical study recently revealed a greater proinflammatory response in females, with significantly increased concentrations of tumor necrosis factor $\alpha$ and IL-6 in the plasma compared with males, when exposed to a bacterial endotoxin. Consequently, a greater proinflammatory response, particularly elevated IL-6 levels, could cause women to experience more severe symptomatology in CHIKV infection, as well as in RA (39).

Smoking also affects men and women differently. The present study demonstrated that male patients that smoked experienced more severe arthralgia in the acute and chronic phases of Chikungunya infection compared with male non-smokers, while female smokers were not significantly different from female non-smokers. Furthermore, in a meta-analysis of 16 studies on patients that smoked, it was identified that male smokers exhibited a summary OR of 1.87 (95\% CI, 1.49-2.34) for developing RA and a summary OR of 3.91 (95\% CI, 2.78-5.50) for positive rheumatoid factor. Furthermore, the same meta-analysis demonstrated that female smokers had respective OR values of 1.31 (95\% CI, 1.12-1.54) and 1.29 (95\% CI, 0.94-1.77) (40). Thus, smoking was a greater risk factor for developing RA in males compared with females, illustrating another similarity between Chikungunya fever and RA.

Patient age was also a significant risk factor in the present study, given that patients $\geq 40$ years of age presented with the highest arthralgia scores. In RA, an adjusted multivariate analysis that included 197 patients demonstrated that late-onset patients presented with greater disease activity (41). This could be associated with an increased concentration of proinflammatory ILs, including IL-6, in the blood of older people (42), which could induce more severe arthralgia in the two diseases. The association between patient age and severity of symptoms is another shared characteristic of Chikungunya fever and RA.

The present study also identified that diabetes and the presence of rash, muscular weakness, headache and conjunctivitis during the acute phase of the disease are associated with the disease course. Likewise, two previous studies have associated diabetes or rash with persistent arthralgia after CHIKV infection $(43,44)$. By contrast, to the best of our knowledge, the occurrence of conjunctivitis during the acute phase as a risk factor for the severity of arthralgia, or headache and muscular weakness as intensity-increasing factors, have not been previously reported.

It is important to emphasize that two different types of statistical analyses were used to interpret arthralgia intensity in the present study: Association of risk factors and comparison of means. The two analyses identified smoking and female sex as relevant risk factors. They were consistent in identifying that a severe acute phase of the disease leads to a severe chronic stage. These variables were the most important in determining the clinical course of the disease. The other symptoms of headache, conjunctivitis, muscular weakness and rash were considered less relevant risk factors, since they were significant in only one statistical analysis. This also implies that the differences between studies on CHIKV infection could be due not only to real differences in disease progression, but also to the type of statistical strategy applied.

A key limitation of the present study was that a single arthralgia intensity score (described by the patient as the most intense pain at the time of the evaluation) was considered for the analysis of arthralgia severity in the chronic phase, regardless of whether such pain was being experienced in $>1$ joint. Furthermore, a multidimensional analysis (RAPID3 scale) that has not appeared in studies in the literature was applied to evaluate the general state of patients during the chronic stage of disease.

It was clear from the results of the current study that smoking, female sex, or having experienced severe arthralgia in the acute phase of CHIKV infection are the primary factors that promote severe sequelae, particularly high-intensity arthralgia. Therefore, it is important to conduct studies that evaluate whether the application of effective antiviral or anti-arthralgia treatments (45) during the acute phase of the disease will reduce the sequelae or severity of infection. In the present study, treatment in the chronic phase was symptomatic and based on NSAIDs. However, the similarity between CHIKV and RA arthralgias has guided some physicians to use methotrexate or anti-tumor necrosis factor agents (standard treatments for RA) in patients with chronic Chikungunya syndrome, with good response and tolerance (46). Despite the success observed in controlled studies, those treatments are not yet recommended in the international clinical management guidelines for patients with CHIKV (47). In the present study, similarities in risk factors for CHIKV arthralgia and RA development were identified or confirmed. Such risk factors are generally supported by common pathophysiological mechanisms, particularly elevated IL-6 levels, so these findings provide a basis for identifying common pathophysiological and therapeutic mechanisms in the two diseases.

The results of the present study could aid physicians in regions that are endemic for CHIKV. The findings prompt more detailed follow-up in female patients and smokers that are infected with the disease, given that they present with a higher risk of intense and chronic illness. At the public health level, these results contribute to a greater understanding of increased symptomatology in infectious diseases in individuals exposed to smoke. The findings provide additional support for strategies that focus on reducing smoke exposure.

In conclusion, smoking and female sex are the key risk factors for presenting with severe joint pain in acute and chronic phases of CHIKV infection. Risk factors for increased severity and chronicity of arthralgia in CHIKV appear to be similar to those associated with the development and severity of RA, which may be due to their shared pathophysiological mechanisms. To the best of our knowledge, this is the first study to associate smoking with the clinical course of CHIKV infection, and therefore more studies are required in this field.

\section{Acknowledgements}

The present study was completed using equipment and resources obtained through grants from the INFRAESTRUCTURACONACYT-2016 (grant no. 270485) and FOSISS-CONACYT2016 (grant no. 272792). 


\section{References}

1. Powers $\mathrm{AM}$ and Logue $\mathrm{CH}$ : Changing patterns of chikungunya virus: Re-emergence of a zoonotic arbovirus. J Gen Virol 88 2363-2377, 2007.

2. Caglioti C, Lalle E, Castilletti C, Carletti F, Capobianchi MR and Bordi L: Chikungunya virus infection: An overview. New Microbiol 36: 211-227, 2013.

3. Goupil BA and Mores CN: A review of Chikungunya virus-induced arthralgia: Clinical manifestations, therapeutics, and pathogenesis. Open Rheumatol J 10: 129-140, 2016.

4. Sepúlveda-Delgado J, Vera-Lastra OL, Trujillo-Murillo K, Canseco-Ávila LM, Sánchez-González RA, Gómez-Cruz O, Lugo-Trampe A, Fernández-Salas I, Danis-Lozano R, ContrerasContreras A, et al: Inflammatory biomarkers, disease activity index, and self-reported disability may be predictors of chronic arthritis after chikungunya infection: brief report. Clin Rheumatol 36 : 695-699, 2017.

5. Kucharz EJ and Cebula-Byrska I: Chikungunya fever. Eur J Intern Med 23: 325-329, 2012

6. Rodriguez-Morales AJ, Villamil-Gomez W, Merlano-Espinosa M and Simone-Kleber L: Post-chikungunya chronic arthralgia: a first retrospective follow-up study of 39 cases in Colombia. Clin Rheumatol 35: 831-832, 2016.

7. Murillo-Zamora E, Mendoza-Cano O, Trujillo-Hernández B, Alberto Sánchez-Piña R and Guzmán-Esquivel J: Persistent arthralgia and related risks factors in laboratory-confirmed cases of Chikungunya virus infection in Mexico. Rev Panam Salud Publica 41: e72, 2017

8. Economopoulou A, Dominguez M, Helynck B, Sissoko D, Wichmann O, Quenel P, Germonneau P and Quatresous I: Atypical Chikungunya virus infections: clinical manifestations, mortality and risk factors for severe disease during the 2005-2006 outbreak on Réunion. Epidemiol Infect 137: 534-541, 2009.

9. van Aalst M, Nelen CM, Goorhuis A, Stijnis C and Grobusch MP: Long-term sequelae of chikungunya virus disease: A systematic review. Travel Med Infect Dis 15: 8-22, 2017.

10. Thanapati S, Ganu M, Giri P, Kulkarni S, Sharma M, Babar P, Ganu A and Tripathy AS: Impaired NK cell functionality and increased TNF- $\alpha$ production as biomarkers of chronic chikungunya arthritis and rheumatoid arthritis. Hum Immunol 78 370-374, 2017

11. Chow A, Her Z, Ong EK, Chen JM, Dimatatac F, Kwek DJ, Barkham T, Yang H, Rénia L, Leo YS and Ng LF: Persistent arthralgia induced by Chikungunya virus infection is associated with interleukin-6 and granulocyte macrophage colony-stimulating factor. J Infect Dis 203: 149-157, 2011

12. Narazaki M, Tanaka T and Kishimoto T: The role and therapeutic targeting of IL-6 in rheumatoid arthritis. Expert Rev Clin Immunol 13: 535-551, 2017

13. Englbrecht M, Tarner IH, van der Heijde DM, Manger B, Bombardier $\mathrm{C}$ and Müller-Ladner U: Measuring pain and efficacy of pain treatment in inflammatory arthritis: A systematic literature review. J Rheumatol 90: 3-10, 2012.

14. Food and Drug Administration: Emergency Use Authorizations. Trioplex real-time RT-PCR assay (CDC), 2016 https://www.fda.gov/MedicalDevices/Safety/EmergencySituations/ ucm161496\#zika. Accessed May 11, 2017.

15. Pincus T, Yazici Y and Bergman MJ: RAPID3, an index to assess and monitor patients with rheumatoid arthritis, without formal joint counts: Similar results to DAS28 and CDAI in clinical trials and clinical care. Rheum Dis Clin North Am 35: 773-778, 2009.

16. Palos GR, Mendoza TR, Mobley GM, Cantor SB and Cleeland CS Asking the community about cutpoints used to describe mild, moderate, and severe pain. J Pain 7: 49-56, 2006.

17. Serlin RC, Mendoza TR, Nakamura Y, Edwards KR and Cleeland CS: When is cancer pain mild, moderate or severe? Grading pain severity by its interference with function. Pain 61: 277-284, 1995

18. Boonstra AM, Stewart RE, Köke AJA, Oosterwijk RFA, Swaan JL, Schreurs KMG and Preuper HRS: Cut-off points for mild, moderate, and severe pain on the numeric rating scale for pain in patients with chronic musculoskeletal pain: Variability and influence of sex and catastrophizing. Front Psychol 7: 1466, 2016.

19. Jones KR, Vojir CP, Hutt E and Fink R: Determining mild, moderate, and severe pain equivalency across pain-intensity tools in nursing home residents. J Rehabil Res Dev, 44: 305-314, 2007.

20. Zelman DC, Hoffman DL, Seifeldin R and Dukes EM: Development of a metric for a day of manageable pain control Derivation of pain severity cut-points for low back pain and osteoarthritis. Pain 106: 35-42, 2003.
21. Aicher B, Peil H, Peil B and Diener HC: Pain measurement: Visual Analogue Scale (VAS) and Verbal Rating Scale (VRS) in clinical trials with OTC analgesics in headache. Cephalalgia 32: 185-197, 2012.

22. Taype-Rondan A, Bernabe-Ortiz A, Alvarado GF, Gilman RH, Smeeth L and Miranda JJ: Smoking and heavy drinking patterns in rural, urban and rural-to-urban migrants: The PERU MIGRANT Study. BMC Public Health 17: 165, 2017.

23. Bondy SJ, Victor JC and Diemert LM: Origin and use of the 100 cigarette criterion in tobacco surveys. Tob Control 18: 317-323, 2009.

24. Tervo L, Mäkelä S, Syrjänen J, Huttunen R, Rimpelä A, Huhtala H, Vapalahti O, Vaheri A and Mustonen J: Smoking is associated with aggravated kidney injury in Puumala hantavirus-induced haemorrhagic fever with renal syndrome. Nephrol Dial Transplant 30: 1693-1698, 2015.

25. Çörtük M, Acat M, Yazici O, Yasar Z, Kiraz K, Ataman SY, Tanriverdi E,Zitouni B, Kirakli C,Ediboglu O, et al: Retrospective review of epidemic viral pneumonia cases in Turkey: A multicenter study. Exp Ther Med 13: 1431-1437, 2017.

26. Groskreutz DJ, Monick MM, Babor EC, Nyunoya T, Varga SM, Look DC and Hunninghake GW: Cigarette smoke alters respiratory syncytial virus-induced apoptosis and replication. Am J Respir Cell Mol Biol 41: 189-198, 2009.

27. McGrath-Morrow S, Rangasamy T, Cho C, Sussan T, Neptune E, Wise R, Tuder RM and Biswal S: Impaired lung homeostasis in neonatal mice exposed to cigarette smoke. Am J Respir Cell Mol Biol 38: 393-400, 2008.

28. Azzopardi D, Haswell LE, Foss-Smith G, Hewitt K, Asquith N, Corke S and Phillips G: Evaluation of an air-liquid interface cell culture model for studies on the inflammatory and cytotoxic responses to tobacco smoke aerosols. Toxicol In Vitro 29: 1720-1728, 2015.

29. Husari A, Shihadeh A, Talih S, Hashem Y, El Sabban M and Zaatari G: Acute exposure to electronic and combustible cigarette aerosols: Effects in an animal model and in human alveolar cells. Nicotine Tob Res 18: 613-619, 2016.

30. Borges ÁH, O'Connor JL, Phillips AN, Rönsholt FF, Pett S, Vjecha MJ, French MA and Lundgren JD, et al: Factors associated with plasma IL-6 levels during HIV infection. J Infect Dis 212: $585-595,2015$.

31. Nadrowski P, Chudek J, Skrzypek M, Puzianowska-Kuźnicka M, Mossakowska M, Więcek A, Zdrojewski T, Grodzicki T and Kozakiewicz K: Associations between cardiovascular disease risk factors and IL-6 and hsCRP levels in the elderly. Exp Gerontol 85: 112-117, 2016.

32. Nakanishi K, Nishida M, Harada M, Ohama T, Kawada N, Murakami M, Moriyama $\mathrm{T}$ and Yamauchi-Takiharaa $\mathrm{K}$ : Klotho-related molecules upregulated by smoking habit in apparently healthy men: A cross-sectional study. Sci Rep 5: 14230, 2015.

33. Sopori ML and Kozak W: Immunomodulatory effects of cigarette smoke. J Neuroimmunol 83: 148-156, 1998.

34. Miner JJ, Aw-Yeang HX, Fox JM, Taffner S, Malkova ON, Oh ST, Kim AHJ, Diamond MS, Lenschow DJ and Yokoyama WM: Chikungunya viral arthritis in the United States: A mimic of seronegative rheumatoid arthritis. Arthritis Rheumatol 67: 1214-1220, 2015

35. Baillet A, Gossec L, Paternotte S, Etcheto A, Combe B, Meyer O, Mariette X, Gottenberg JE and Dougados M: Evaluation of serum interleukin-6 level as a surrogate marker of synovial inflammation and as a factor of structural progression in early rheumatoid arthritis: Results from a French national multicenter cohort. Arthritis Care Res (Hoboken) 67: 905-912, 2015.

36. Van Keulen HV, Gomes AS, Toffolo MC, Oliveira EE, Silva LC, Alves CC, Almeida CS, Dutra SC, Aguiar AS and Ferreira AP: Serum levels of nitric oxide and cytokines in smokers at the beginning and after 4 months of treatment for smoking cessation. Int J Cardiol 230: 327-331, 2017.

37. van Aalst M, Nelen CM, Goorhuis A, Stijnis C and Grobusch MP: Long-term sequelae of chikungunya virus disease: A systematic review. Travel Med Infect Dis 15: 8-22, 2017.

38. Svensson B, Andersson M, Forslind K, Ajeganova S and Hafström I; BARFOT study group: Persistently active disease is common in patients with rheumatoid arthritis, particularly in women: a long-term inception cohort study. Scand J Rheumatol 45: 448-455, 2016.

39. Wegner A, Benson S, Rebernik L, Spreitzer I, Jäger M, Schedlowski M, Elsenbruch S and Engler H: Sex differences in the pro-inflammatory cytokine response to endotoxin unfold in vivo but not ex vivo in healthy humans. Innate Immun 23: 432-439, 2017. 
40. Sugiyama D, Nishimura K, Tamaki K, Tsuji G, Nakazawa T, Morinobu A and Kumagai S: Impact of smoking as a risk factor for developing rheumatoid arthritis: a meta-analysis of observational studies. Ann Rheum Dis 69: 70-81, 2010.

41. Ruban TN, Jacob B, Pope JE, Keystone EC, Bombardier C and Kuriya B: The influence of age at disease onset on disease activity and disability: Results from the ontario best practices research initiative. Clin Rheumatol 35: 759-763, 2016.

42. Sebastiani P, Thyagarajan B, Sun F, Honig LS, Schupf N, Cosentino S, Feitosa MF, Wojczynski M, Newman AB, Montano M and Perls TT: Age and sex distributions of age-related biomarker values in healthy older adults from the long life family study. J Am Geriatr Soc 64: e189-e194, 2016.

43. Schilte C, Staikowsky F, Couderc T, Madec Y, Carpentier F, Kassab S, Albert ML, Lecuit M and Michault A: Chikungunya virus-associated long-term arthralgia: A 36-month prospective longitudinal study. PLoS Negl Trop Dis 7: e2137, 2013.
44. Yaseen HM, Simon F, Deparis X and Marimoutou C: Identification of initial severity determinants to predict arthritis after chikungunya infection in a cohort of French gendarmes. BMC Musculoskelet Disord 15: 249, 2014.

45. Abdelnabi R, Neyts J and Delang L: Chikungunya virus infections: Time to act, time to treat. Curr Opin Virol 24: 25-30, 2017.

46. Blettery M, Brunier L, Polomat K, Moinet F, Deligny C, Arfi S, Jean-Baptiste $\mathrm{G}$ and De Bandt M: Brief report: Management of chronic post-chikungunya rheumatic disease: The martinican experience. Arthritis Rheumatol 68: 2817-2824, 2016.

47. World Health Organization: Guidelines on Clinical Management of Chikungunya Fever. http://www.wpro.who. int/mvp/topics/ntd/Clinical_Mgnt_Chikungunya_WHO_SEARO. pdf. Accessed Oct 10, 2017. 